\title{
The Study of Application of Microscopic Material Form in Comprehensive Material Painting
}

\author{
Yishan Deng \\ South China University of Technology \\ Guangzhou, China 510000
}

\author{
Weihai Zhang* \\ South China University of Technology \\ Guangzhou, China 510000 \\ *Corresponding Author
}

\begin{abstract}
Microscopic materials cannot be seen with the human eye, they are invisible matters which can only be observed by advanced scientific apparatus. The emergence of the art of comprehensive material painting was in last century in West, it has developed a lot after it entered China. The manners of comprehensive material painting becoming more various and its artistic showing ability was getting strong at the development of times, the publishing of more and more diversified comprehensive material painting compositions sufficiently represented artistic spirits of those New-era painters. Using the concept of microscopic material form throws a light in composing comprehensive material painting. This paper aims to discover the properties of microscopic material form such as aesthetic and visual values which are always neglected by people; we are trying to apply microscopic material form in artistic composition in order to make microscopic form of microscopic science more fitted in comprehensive material painting. In this way we can know more about different aspects of microscopic material so as to give more attention to science, in the meantime, it could also help us to expand our material source of comprehensive painting effectively.
\end{abstract}

Keywords-microscopic material form; comprehensive material; painting; art

\section{INTRODUCTION}

Art varies as time goes by and its trend of diversity becomes more and more obvious. The appearance of advanced technology brings us a new thing - the microscopic material. Microscopic material has various forms and different characteristics, those relations exist in traditional aesthetics such as lightness and darkness, points and lines are also exist in microscopic world. The elements in these relations are themselves opposite to each other in macro world, but in micro world, there are always infinite possibilities, for micro world has specific artistic features which macro world doesn't have. Artists apply advanced technologies in their composition by combine science and art together and the work created in this manner is called Micro Art. Besides, each material has its own character of which artists make use to start their researches, they put them into practice and thereby the materials representing various visual effects or spirit connotation. Comprehensive material painting differs traditional painting in their ways of emotional expression, yet it merges with contemporary art and thus becomes more modernistic. In comprehensive painting art, the expressive ability of material art has been approved of by more and more artists nowadays and the development of this ability brings more possibilities for the creation of modern arts.

\section{MiCROSCOPIC MATERIAL FORM}

When advanced scientific apparatus such as transmittedlight microscope, scanning electron microscope, medical instrument haven't been invented, people can only observe nature in general ways, yet people finally realized the micro world since these apparatus been invented, this realization deepening the knowledge about nature which accelerates the process of art creation. There exist two ways to study and research about micro material art creation: one is to use micro scientific technology to create art work, the other is to combine micro science and art together. The latter way is the mainly point in this paper. We will study and analyze the artistic features of microscopic material form in aesthetic way.

\section{A. The Concept of Microscopic Material}

Microscopic materials cannot be seen with the human eye, they are invisible matters which can only be observed by advanced scientific apparatus. In general, those minute matters cannot be sensed by human organs are called "microscopic objects", they mainly consist the micro world.

\section{B. The Categories of Microscopic Material Form}

1) Cell: The cell is the basic structural and functional unit of all known living organisms. Cell consists of several types, animal cell, plant cell, etc. Cell is the most popular subject for medical and biological research, and it has been used widely in picture information and art creativity. Cell performs different structure formation by amplification and colorization. In all living organisms, cells stringed together tightly but each has its own independence and orderly stated.

2) Microorganism: Microorganism has many forms, virus and bacteria are most seen as poisonous so that people always keep way from them, but blankly speaking, people cannot live without virus and bacteria. Bacteria distributed most widely in all organisms, they are categorized into following types: bacillus, coccus, etc. Virus is a non-bacteria organism. It has many forms such as filament and brick. 
3) Crystal: Crystal is a solid material consists of flats with specific orientations and its constituents are arranged periodically and in highly ordered microscopic structure. Generally, different kinds of crystal has different structure such as hexahedron and cube, they are all symmetrical. Rocks, salts are crystals in micro vision.

4) Others: Examples above are just several typical type in micro world, not the whole, there are still other types such as furs, drug's ingredient, etc.

\section{COMPREHENSIVE MATERIAL PAINTING}

\section{A. The Introduction of Comprehensive Material Painting}

Comprehensive material painting is different from traditional material painting in modern art. It refers to some novel approaches to create artistic work, for instance, glues sack bag and metal material to the picture and then paint it, and it is more like static painting which contained the drawing technology.

Comprehensive material painting's appearance breaks the limit between different paintings types, painting expressiveness transform from mono-type to multi-type of visual effect. China faces a severe challenge in arts, the development situation will surely change because of the strongly crash brought by western-type arts, in art work creation, its expressional manner - comprehensive material breaks the limit of traditional material's expressiveness and enlarges the performance space of drawing art, with the use of its specific physical, cultural and spiritual features.

\section{B. The Character of Comprehensive Material Painting and Its Performance in Drawing}

Comparative to traditional material painting, the advantage of comprehensive material painting is that it can fully represent its texture characteristic and its veining features; traditional material painting doesn't have this expressive ability and tension. Since each material has different character, one can exert this ability and express the strong emotion that he wants only if he apprehends these materials deeply. Generally speaking, painting comprehensive material has two types: basic materials and creative materials.

First, basic materials containing many materials, such as woods, canvas, plastic foam, etc. The different basic materials you use, the different result will be since their different characteristics. Drawing paper and canvas distinct in their water absorbing ability and quality, so is their performance in veining results with pigment. Plastic foams have strong durability, it can be used for painting through specific ways (cut, grub, etc.) to promote their dimensionality, and it is an auxiliary expression manner in art creativity. Using building materials (plaster, glass, etc.) as basic materials to process drawing can perform a stronger sense-of-times than traditional paintings. Making use of chemical materials for basic materials can extend painting's spaciousness and imagery... Artists should choose monotype or multi-type of basic materials according to their demands, providing appropriate condition for subsequent creativity by shaping flat or cubic painting basement.
Second, comparative to traditional painting, comprehensive material painting has more materials to be chosen of, it revised the traditional painting manner which uses only one type of material and makes image more emotional. Some western materials mixes propylene and oil pigment together so as to enlighten the brightness and enrich the color of painting, besides the application of propylene can make the painting more solid. The combination of multiple colors makes the painting more contemporary and modernistic, providing more spaces for artists to compose freely. Apart from the pigment, other aspects of each kind of painting also can represent the expressive ability of comprehensive material paintings.

\section{HOW TO APPLY MiCROSCOPIC MATERIAL FORM IN COMPREHENSIVE MATERIAL PAINTINGS}

\section{A. The Re-creation of Microscopic Material Form}

1) Extract its form: "Form" contains all the outside shape and inner structure of an object except its spatial position and orientation, it also be seen as an object's spatial shape. The "form" has a specific aesthetic character in microscopic material form, some of its form even need not any procession or modification, the micro material picture below gives us an intact structure sense, yellow and purple mainly consist this picture, the dense rays and waves represent a metallic feel leisure but deliberate. One can hardly imagine that this is a crystal picture of vitamin $h$ which was amplified 10 times by transmitted-light microscope. As shown in "Fig. 1", from aesthetic aspect, it is a perfect microscopic material painting without any modification.

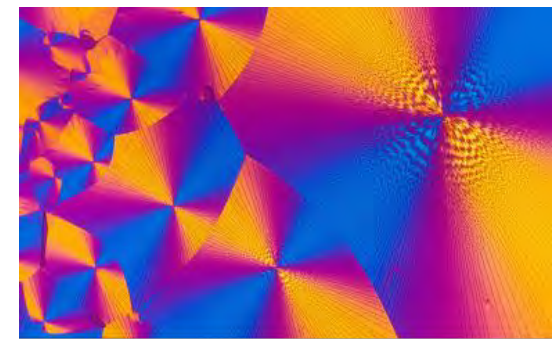

Fig. 1. The figure source www.vcg.com.

Copying directly cannot mix microscopic material into comprehensive material paintings, it is because microscopic material image has to be modified in most situation, in other words, one must apprehend the concept of "form" totally so that to create new image with "form" as its basement. This manner not only maintains the creativity and novelty of composition, but also can mix the concept of aesthetics into it then make the composition more sense of century and graphic design.

2) The apprehension of its meaning: The deep meaning of microscopic material can represent by physical matter, but this representation doesn't come out easy. The outing of the inner meaning often demands the concentration and the extraction of the "form" in micro material in order to know more about its inner world, after extraction, one should 
recreate the information of inner world, then mix the "meaning" into the creation. By the way, the creation doesn't mean one can do anything he likes, it requires that the artist should perform his wants rightly and shows his specialty, then, unfold the lavish language of his work to people.

As shown in "Fig. 2" and "Fig. 3", we used a mouldy potato as the micro material to create a comprehensive material painting. The material is only a potato, but we can find a microscopic world through the amplify observation of a small part around the mouldy lump of this potato. So, we abstract the "form" of the mouldy lump in this potato, then give it a new inner sense, finally, we integrate this sense in our paintings to create a new composition with deeper spirit.

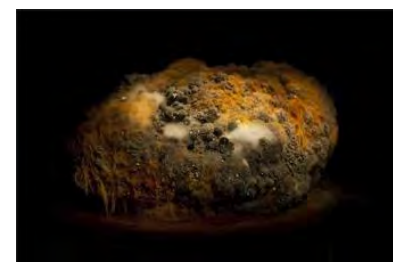

Fig. 2. Figure source www.3jrx.com.

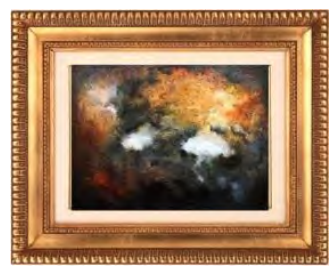

Fig. 3. Microscopic - Yishan Deng.

3) The mastery of its color: The direct and fastest crash to human eyes in a picture is color. The rightful mastery of color can transmit its meaning to viewer better. In psychology, people has strong feelings about color, color might has direct or indirect impact on people's aesthetic ability, characteristics and emotions. Inappropriate application of color in a picture not only affects the accuracy of people's sensation, but also makes against performance of other elements. Colorizing the microscopic material image to make people feel comfortable and natural is not a rare thing. The advanced technology now can follow the color application rule to harmonize the different color, in order to make a perfect balanced colored picture. Studying these new technologies can make us create more specific art works and to catch people's eyes.

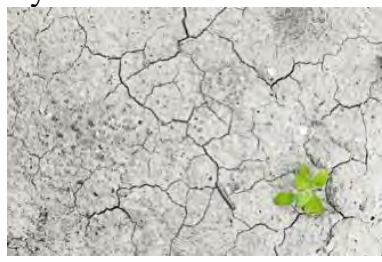

Fig. 4. Figure source www.58pic.com.

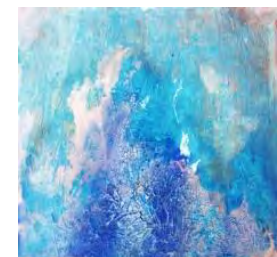

Fig. 5. "Experimental Work 1" - Yishan Deng.

As shown in "Fig. 4", a sun crack wall soaked by the raining water, we capture a part of its texture pattern and colorize it with the brightness of the microscopic element, and then we can create a chromatic piece of art, see "Fig. 5". The colorization work can make more specific impression on human eyes and heart than the original material.

\section{B. The Application of Microscopic Material Form in Comprehensive Material Paintings}

It is said that the beauty of drawing art is that it is and it isn't. The core of this art is the use of abstract and figurative concept and the approaches would be changed as the creator's ideas differ.

1) Figurative method: Figurative method refers to use concrete matter as object to process creation. It usually doesn't change much about relative elements but use original structure as the "form". The use of figurative method mainly occur in realistic drawing about botanic and microorganisms, this method aims to make the viewer know the true meaning of this work by arising the emotional feeling and connecting in mind. In our daily life, this is not rare. One designer uses high frame rates flies pictures as the cover of camera's ads. Such image would make people associate the camera with his mind and to have some interests in the product. Another example is that the severely damaged lungs are always be used as the cover in anti-smoking ads, the ads make a strong impact on audient's mind so as to warn people of the harm smoking would make.

We use figurative method to finish the image processing with this "Fig. 6" of jellyfish living in the deep sea, after the process, we obtain the work as this "Fig. 7". In this process, we combine the jellyfish with a half body of a dancing dancer, in the method we reserve the "form" of the jellyfish, yet in the next procedure we use turpentine, acrylic paint, white emulsion and tissue, etc. With the help of these materials, we keep breaking the "form" and discover a new "form", in the end, we basically retain the figurative formation of the jelly fish and the composition is complete.

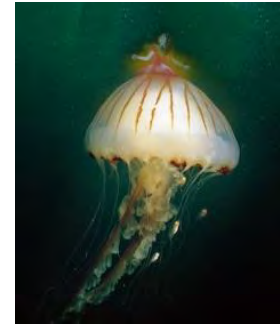

Fig. 6. Figure source www.nipic.com. 




Fig. 7. "Jellyfish" - Yishan Deng.

2) Abstract method: The purpose of abstract method is to extract multiple features and to simplify the concept it contains, which would help people to know about the meaning of it more easily. Abstract method doesn't depict the matter itself, its aesthetic rules are not accomplished, yet the abstract painting always gives people a feeling of natural and realistic. Two different concepts are used in managing the abstractive micro material form. One is to manage in an abstractive way with the elements of image itself; another is that the artist revises the elements in artistic way. Actually, the beauty is contained in painting's artistic effect and its cultural background, so it is necessarily that artist should maintain the scientific side of microscopic material form when represents the artistic side, it is wrong for artist to exaggerate the artistic side thus ignore the cultural beauty underlying the surface, this isolation would only make the composition worse. The beauty that produced through the composition doesn't possess realistic meaning, yet it deepen the relation between different people and different matters, the artist should use their imagination to put the concentration on recreation of the elements structure in microscopic material form, reserve his own specific features and beauties, see "Fig. 8" and "Fig. 9".



Fig. 8. Figure source www.58pic.com.



Fig. 9. "Experimental Work 2" —Yishan Deng.

We discover the specific part with the texture element in the bark of a trunk and present it in the image with the abstract method, we don't process these elements with the simple method of copy and paste or just describe its form with figuration, and instead we process with the texture elements in bark artistically. We can both reserve the beauty of elements and can make the features of the texture part in bark more obvious.

\section{CONCLUSION}

Micro science is a new creative field which based of micro technologies. It is invented under the development of times. Along with the expedition in micro science, micro world is known by more and more people, this expand people's knowledge of our living world. Microscopic material for is definitely a new matter comparative to macro world which is realized by people for thousands of years, so many artists have put their hearts in microscopic world, they fulfill their imagination ability and creativity to apply new elements in micro world to artistic creation field, it is not only the enlargement of the expressional ways, but also make comprehensive material paintings more emotional and inflectional.

The micro world has been known by people gradually nowadays. People continuously explore the micro world by photography, paintings and other artistic ways. This exploration is good for people to cultivate their artistic creative and their cognitive ability and thus can form a favorable society circumstance which can drive people to learn more about scientific stuff. The constant learning and adventuring in micro world by this whole society propels the integration of science and art, one day micro scientific arts would become a new genre with the combination of these two elements, and this new genre would be the brightest star in the process of art and scientific development.

\section{REFERENCES}

[1] Kuang Yingfeng. On the definition of micro art [J]. art education research, 2018 (11): 60

[2] Deng Chenxi. Research on aesthetics and painting performance of materials in comprehensive material painting [J]. arts and technology, 2017,30 (03): 226

[3] Zhang Liangyu. Application of micro material form in painting creation [D]. South China University of Technology, 2017.

[4] Zhang Liangyu. The infinite possibilities brought by the micro-world to artistic creation - Appreciation of Clary Reith's Cellular Painting Series [J]. Meiji Times (Middle), 2017 (03): 80-81.

[5] Li Huixue. On the artistic expression of composite materials in painting [J]. art education research, 2014 (16): 19. 